\title{
ON AN INVERSE FORMULA OF A TRIDIAGONAL MATRIX
}

\author{
TOMOYUKI SUGIMOTO
}

Abstract. This paper provides an inverse formula freed of determinant expressions for a general tridiagonal matrix. This is viewed as an alternative version of the Usmani formula, which easily tends to blow up computationally. We discuss a number of different viewpoints regarding the proposed and Usmani's formulas, such as the proof method and the meaning of included terms, although our formula itself may be obtained by a simple transformation of Usmani's. A study of the limit elements based on the inverse formula and a numerical experiment for comparison with the other inverse methods are provided. In addition, we briefly discuss the inverse formula in the case of zero minors, which is illustrated by a numerical example.

Mathematics subject classification (2010): 15A09, 15A06.

Keywords and phrases: Tridiagonal matrix, inversion, product integral, Volterra equation, computational stability, limit elements, zero minors.

\section{REFERENCES}

[1] Q. AL-HASSAN, An algorithm for computing inverses of tridiagonal matrices with applications, Soochow Journal of Mathematics 31 (2005), 449-466.

[2] W. W. BARRETT, A theorem on inverses of tridiagonal matrices, Linear Algebra and its Applications 27 (1979), 211-217.

[3] M. E. A. El-Miknawy, On the inverse of a general tridiagonal matrix, Applied Mathematics and Computation 150 (2004), 669-679.

[4] M. EL-MiKKAWY AND A. KARAWIA, Inversion of general tridiagonal matrices, Applied Mathematics Letters 19 (2006), 712-720.

[5] M. A. El-Shehawey, G. A. El-Shreef And A. S. Al-Henawy, Analytical inversion of general periodic tridiagonal matrices, Journal of Mathematical Analysis and Applications 345 (2008), 123134.

[6] J. W. LEWIS, Inversion of tridiagonal matrices, Numerische Mathematik 38 (1982), 333-345.

[7] H.-B Li, T.-Z. HUANG, X.-P. LiU AND H. LI, On the inverses of general tridiagonal matrices, Linear Algebra and its Applications 433 (2010), 965-983.

[8] R. GiLl And S. Johansen, Survey of product-integration with a view toward application in survival analysis, The Annals of Statistics 18 (1990), 1501-1555.

[9] R. J. HANSON, A cyclic reduction solver for the IMSL Mathematics Library, IMSL Technical Report 9002, IMSL, Houston, 1990.

[10] Y. HuAng AND W. F. MCColl, Analytical inversion of general tridiagonal matrices, Journal of Physics A: Mathematical and General 30 (1997), 7919-7933.

[11] R. K. MALLIK, The inverse of a tridiagonal matrix, Linear Algebra and its Applications 325 (2001), 109-139.

[12] R. RAn, T. HUANG, X. LiU AND T. GU, An inversion algorithm for general tridiagonal matrix, Applied Mathematics and Mechanics 30 (2009), 247-253.

[13] G. SimONS AND Y.-C. YAO, Approximating the inverse of a symmetric positive definite matrix, Linear Algebra and its Applications 281 (1998), 97-103.

[14] T. Sugimoto, Asymptotic distribution of the nonparametric distribution estimator based on martingale approaches in doubly censored data, unpublished manuscript, 2010.

[15] T. Sugimoto, Wald-type variance estimation for the nonparametric distribution estimators in doubly censored data, Annals of the Institute of Statistical Mathematics 63 (2011), 645-670. 
[16] R. Usmani, Inversion of Jacobi's tridiagonal matrix, Computers \& Mathematics with Applications 27 (1994), 59-66.

[17] R. USMANI, Inversion of a tridiagonal Jacobi matrix, Linear Algebra and its Applications 212/213 (1994), 413-414. 